Nature. 2014 January 2; 505(7481): 35-36. doi:10.1038/nature12844.

\title{
The beginning of the end
}

\author{
JUDITH CAMPISI \\ Buck Institute for Research on Aging, Novato, California 94945, USA, and at the Lawrence \\ Berkeley National Laboratory, Berkeley, California.
}

\begin{abstract}
Studies in mice and humans suggest that cellular senescence, the cessation of cell proliferation that is known to suppress cancer and promote ageing, may have evolved to regulate embryonic development.
\end{abstract}

Cells that experience certain types of stress, particularly stress that is potentially cancercausing, undergo an essentially permanent arrest of proliferation termed cellular senescence ${ }^{1}$. Since its formal description in the 1960s, cellular senescence has been thought both to suppress the development of cancer and to promote ageing. Support for these roles has come from tumour studies in mice and humans ${ }^{2}$, and from the realization that senescent cells secrete proteins that cause inflammation, a hallmark of ageing tissues ${ }^{3}$. More recently, a complex inflammatory response called the senescence-associated secretory phenotype (SASP) was shown to facilitate tissue repair and remodelling, and to help the immune system recognize and eventually remove senescent cells ${ }^{4}$. These multiple functions of cellular senescence are not mutually exclusive, but they raise an interesting teleological question: for what purpose did senescence evolve? Findings by Muñoz-Espín et al. ${ }^{5}$ and Storer et $a l .{ }^{6}$, published in Cell, suggest a surprising answer: to fine-tune embryogenesis.

Both research groups found evidence for the presence of senescent cells in mouse and human embryos. To identify these cells, the researchers initially relied on a commonly used marker of senescence, the activity of an enzyme known as senescence-associated $\beta$ galactosidase (SA- $\beta$-gal). Their combined results identified non-dividing SA- $\beta$-galcontaining cells in the embryonic kidney, the endolymphatic sac of the inner ear, developing limbs, the closing neural tube and the apical ectodermal ridge, among other structures. Further analyses showed that non-dividing cells in these structures also expressed high levels of p21, a cell-cycle-inhibitor protein that is often expressed by senescent cells in culture and in postnatal tissues, and of a subset of SASP proteins, which are presumed to facilitate the infiltration of immune cells and eventual clearance of senes-cent cells (Fig. 1).

Surprisingly, however, both groups found that non-dividing cells in these embryonic structures did not express p16 ${ }^{\mathrm{INK} 4 \mathrm{a}}$, a cell-cycle-inhibitor and tumour-suppressor protein that is commonly produced by senescent cells in culture and in postnatal tissues; instead, they expressed p15, another cell-cycle inhibitor that is produced by only some non-

(C) 2013 Macmillan Publishers Limited. All rights reserved jcampisi@buckinstitute.org. 
embryonic senescent cells. Similarly, the cells showed no evidence of a DNA-damage response or activation of $\mathrm{p} 53$, the tumour-suppressor and transcriptional-regulator protein that controls the senescence response to tissue damage or cancer-causing stress. The authors also show that senescence in the embryo depended on $\mathrm{p} 21$, whereas senescence in nonembryonic tissues depends primarily on p53 and p16 ${ }^{\text {INK4a }}$. Moreover, p21 expression in the embryo was induced by two transcription factors, FOXO and SMAD, which are controlled by the PIK and TGF- $\beta$ signalling pathways; by contrast, induction of $\mathrm{p} 21$ during nonembryonic senescence is generally mediated by the DNA-damage response and p53. Thus, the senescence that occurs in embryos shares some, but not all, features of the senescence responses that suppress cancer and facilitate tissue repair (Fig. 1).

What functions do senescent cells serve in the embryo? The authors of both papers speculate that the cells might fine-tune the development of tissue structures in the embryo, as proposed 20 years ago ${ }^{7}$. In addition to curtailing their own proliferation, senescent cells secrete factors that have potent effects on other cells ${ }^{4}$, including effects on apoptotic cell death, cell migration, immune-cell infiltration and angiogenesis (the generation of new blood vessels). It was surprising, therefore, that the researchers found only a few pre- or post natal abnormalities in mouse embryos rendered senescence-free by deletion of the gene encoding p21. Of course, embryos are remarkably plastic and, indeed, the authors' analyses of the kinetics and structure of morphogenesis in the senescence-free embryos showed that other tissue-remodelling processes largely compensate for the lack of senescence.

The results reported by Muñoz-Espín et al. and Storer $e t$ al. are consistent with their view that cellular senescence evolved to optimize embryogenesis, and that its beneficial post-natal functions (tumour suppression and tissue repair) arose later during evolution. However, the distinct but overlapping manifestations of senescence in embryonic and postnatal tissues need not be a consequence of sequential evolution. Rather, cells might be programmed to link arrested cell proliferation to other cellular responses, including a secretory phenotype, to meet a variety of physiological needs and respond to various forms of stress. This possibility would explain why some senescent states seem to depend primarily on $\mathrm{p} 53$, others on $\mathrm{p} 16^{\mathrm{INK} 4 \mathrm{a}}$, yet others on $\mathrm{p} 21$, and so on. It might also explain why there are no markers that are unique to senescent cells ${ }^{4}$. Finally, the idea that senescence responses are assemblies of cellular characteristics might explain why the SASP proteins differ depending on the senescence inducer, cell type and tissue of origin ${ }^{8}$, including whether the senescent cells reside in post-natal tissues or the embryo ${ }^{5,6}$.

Regardless of the origin of cellular senescence, the deleterious (pro-ageing) effects of senescent cells are clearly maladaptive. The number of senescent cells increases with age in many tissues, possibly because they are incompletely eliminated by the immune system and/or produced in greater numbers in aged organisms. Consequently, aged tissues might suffer from an accumulation of non-dividing cells and the persistent presence of SASP factors that can promote chronic inflammation and alter tissue structure and function.

The findings that vertebrate embryos are replete with cells bearing characteristics of senescent cells opens up possibilities for furthering our understanding of the relationship between embryonic and adult cells, and how tissue regeneration, tumour suppression and 
ageing are balanced. They also raise ideas for potential therapies. Is it possible, for example, to activate embryonic senescence programs to optimize tissue repair postnatally or even in aged adults? Answers to such questions depend, of course, on future research.

\section{References}

1. Campisi J, d’Adda di Fagagna F. Nature Rev. Mol. Cell Biol. 2007; 8:729-740. [PubMed: 17667954]

2. Collado M, Serrano M. Nature Rev. Cancer. 2010; 10:51-57. [PubMed: 20029423]

3. Cevenini E, Monti D, Franceschi C. Curr. Opin. Clin. Nutr. Metab. Care. 2013; 16:14-20. [PubMed: 23132168]

4. Rodier F, Campisi J. J. Cell Biol. 2011; 192:547-556. [PubMed: 21321098]

5. Muñoz-Espín D, et al. Cell. 2013; 155:1104-1118. [PubMed: 24238962]

6. Storer M, et al. Cell. 2013; 155:1119-1130. [PubMed: 24238961]

7. Martin GM. J. Gerontol. 1993; 48:B171-B172. [PubMed: 8366255]

8. Coppé J-P, et al. PLoS Biol. 2008; 6:e301. 


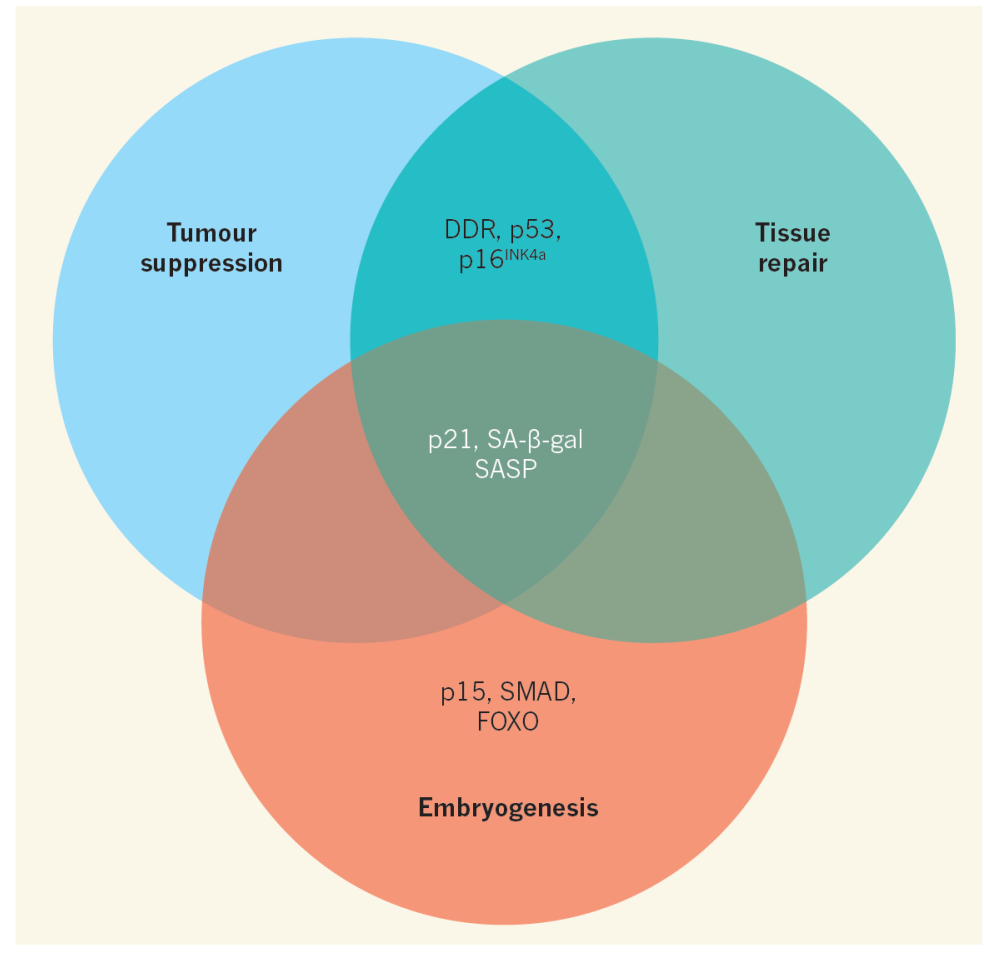

Figure 1. Senescence modules

Cellular senescence is involved in tumour suppression, tissue repair and, as shown by Muñoz-Espín et al..$^{5}$ and Storer et al. ${ }^{6}$, embryogenesis. In all three cases, senescent cells express the protein $\mathrm{p} 21$ and proteins associated with the senescence-associated secretory phenotype (SASP); they also exhibit senescence-associated $\beta$-galactosidase (SA- $\beta$-gal) activity. However, during tumour suppression and tissue repair, senescence depends primarily on the activity of the proteins $\mathrm{p} 53$ and $\mathrm{p} 16^{\mathrm{INK} 4 \mathrm{a}}$, which are often induced as part of the DNA-damage response (DDR). By contrast, senescence during embryogenesis depends on p21, which is switched on by the transcription factors FOXO and SMAD. Senescent cells in the embryo also express p15. 